druckmessung bestimmten sein, z. B. bei Glykogen, dessen Molekulargewicht nach Bestimmung der Gefrierpunkts-Erniedrigung durch Gatin-Gruzewska ${ }^{8}$ ) die Löslichkeit in Wasser vorausgesetzt … über 140000 sein müßte; Versuche, die ich in Gemeinschaft mit H. Kasarnowski angestellt habe, zeigten deutliche Diffusion, wenn auch vorläufig leider die Messung der Diffusionskonstanten durch die Verunreinigung der Präparate mit Dextrinen unmöglich war. Bei Ovalbumin erhielten wir dagegen mit Hilfe der Diffusion einen Wert von ca. 20000 für das Molekulargewicht, was gut übereinstimmt mit dem von C. J. Martin ${ }^{9}$ ) ermittelten Wert von $20-30000$, und der Größenordnung nach mit dem Wert für Hämoglobin, den E. W. Reid 19) zu 48000 gefunden hat. Es ist natürlich nötig, diese Messungen weiter auszudehnen. -- Prinzipiell ist noch eine dritte Methode zur Orientierung über das Molekulargewicht der Kolloide denkbar, die Bestimmung der Oberflächenspannung ${ }^{11}$ ); doch ist sie hoffnungsloser als die erste Methode, da die Oberflächenspannung bekannitlich durch kleine Mengen von Fremcstoffen, von denen die Kolloide eben schwer trennbar sind, stark beeinflußt wird.

Ist also schon die Sicherheit der Größe des Molekulargewichtes nicht gar groß, so liegt eine weitere Schwierigkeit in ihrer Beziehung
8) Pflüg. Arch.
Bd. 103, S. 282 (1904)
C. $R$.

Bd. 138 , S. 1631 .

9) Journ. of physiol. Bd. 20, S. 364 cit. nach

H. A ron. Bioch. C. Bd. 3, S. 509 (1905).

10) Journ. of physiol. Bd. 33, S. 13 (1905). s. 1375 . auf die Teilchengröße. Nach den Versuchen von Linder und Picton ${ }^{12}$ ) scheint die Teilchengröße wenigstens gewisser Kolloide von ihrer Herstellung und vielleicht von anderen Verhältnissen, kurz von der Vorgeschichte abhängig zu sein, dasselbe lehren die Versuche von Zsigmondy ${ }^{13}$ ), F. Krafft 14), Kahlenberg und Schreiner ${ }^{15}$ ), sowie anderer. Ferner zeigt auch das Ultramikroskop ${ }^{16}$, daB stets ein Gemisch von sehr verschieden großen Teilchen vorliegt. Wird aber das Molekulargewicht etwa durch die mittlere Teilchengröße bestimmt? Dabei hat man noch keinerlei Orientierung darüber, ob etwa eine GesetzmäBigkeit zwischen den Mengen der verschieden großen, in der Lösung befindlichen Teilchen besteht ${ }^{17}$ ) (z. B. im Sinne eines chemischen Gleichgewichts) oder ob allein Oberflächenkräfte maßgebend sind oder beides.

Jedenfalls ist die Beziehung zwischen Molekulargewicht (osmotischem Druck) und Teilchengröße eine noch völlig ungeklärte Frage der Kolloidchemie ${ }^{18}$ ).

Chemisches Institut d. Techn. Hochschule, Karlsruhe.

12) Journ. of the chem. Soc. Bd. 61, S. 148 (1892).

19) Vgl. Zur Erkenntnis der Kolloide. Jena. 1905.

14) Ber. d. D. chem. Ges. Bd. 28, S. 2573 (1895), Bd. 29, S. 1328 (1896), Bd. 32, S. 1584 (1899)

15) Ztschr. f. physik. Chem. Bd. 27, S. 552 (1898). Das. auch A. Smits. Bd. 45, S. 608 (1903).

16) Vgl. Zsigmondy l. c. ferner z. B. Raehlman n, Münchn. med. Wochenschr. Bd. 50, S. 2089 (1903).

$\left.{ }^{17}\right)$ Vielleicht gelingt es mit Hilfe der Diffusionsstudien, einige Âuflärung über diese Fragen zu gewinnnen.

18) Nach AbschluB dieser Notiz finde ich Andeutungen ähnlicher Art im eben erscheinenden Lehrbuch der physik. Chemie v. F. W. Küster, S. 296 (1907).

\title{
Die Gallertfiltration.
}

Von Dr. H. Bechhold.

Im Oktober vorigen Jahres brachte die "Zeitschr. f. Kolloidchemie eine kurze Notiz über "Fraktionierte Filtration von Kolloiden ", in der ich mitteilte, daß sich Gallerten ausgezeichnet als Filter verwenden lassen und daß sie je nach ihrer Konzentration mehr oder minder durchlässig sind.

Ich war nun der Ueberzeugung, daß der Gedanke der Gallertfiltration ein sehr fruchtbarer werden könnte und daß es nur der Ausbildung der Methode bedürfte, um sie zu einem allgemeinen Hilfsmittel des Chemikers, insbesondere des Kolloidchemikers, zu machen. Ich glaube behaupten zu dürfen, daß mir dies ge- lungen ist, ${ }^{1}$ ) so daß es jedermann möglich ist, die leicht handhabbare Methode für praktische oder wissenschaftliche $Z$ wecke zu verwenden. Sie erlaubt bei ganz geringen Überdrucken $(0,2$ bis 5 Atmosphären) durch ein äußerst billiges, in Massen herstellbares, sterilisierbares Material eine rasche Filtration $z \mathfrak{u}$ erzielen. Auch meine Voraussetzung erwies sich als richtig, daB es nämlich möglich sein müsse, die Filterdichte beliebig zu variieren, indem man die Gallertkonzentration abãndert.

1) Die genauen Zahlenangaben und Einzelheiten nebst Literaturnachweisen erscheinen demnächst in der "Zeitschr. ?. physik. Chemie". 
Als Gallerten benutzte ich hauptsächlich Eisessigkollodium, das durch Eintauchen in Wasser gelatiniert war, ferner Gelatine, die in Formaldehyd gehärtet wurde. Durch die Tecknik der künstlichen Seide sind uns jedoch noch so viele andere Substanzen in die Hand gegeben, daß kein Mangel an verschiedenartigem Filtermaterial vorliegt.

Um der Gallerte einen Halt zu geben, muß man Gewebe, Filterpapier oder dergleichen damit imprägnieren, wobei sich der Gedanke von Geh. Rat Ehrlich, nämlich die Verwendung dünner Gallertschichten als sehr zweckmäßig erwies.

In den meisten Fällen zeigte es sich als das praktischste, starkes, rauhes Filterpapier mit der Gallerte zu imprägnieren. Taucht man dasselbe in die Gallertlösung, so bleiben häufig Luftblasen zurück und die Gallerte bildet unter Umständen nach dem Gelatinieren nur eine leicht verletzbare äuBere Haut, so daß viele Filter unbrauchbar sind. Um diesem Uebelstande zu begegnen, habe ich später die Filter im Vakuum imprägniert. Ich benutze dazu einen Apparat Fig. 1. Auf den rechteckigen Glastrog $\mathrm{T}$ ist

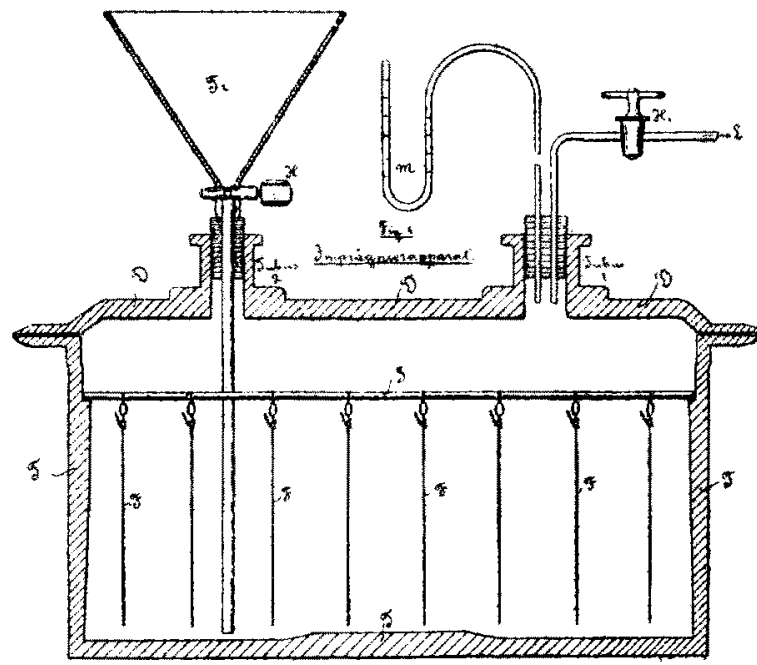

Fig. 1.

der Deckel D luftdicht aufgeschliffen. An der Querstange S sind eine Anzahl runder Filterscheiben $F$ (in meinen Apparat gehen ca. 12) aufgehängt. Der Deckel D hat zwei Tuben. Durch Tube 1 gehen zwei Röhren; die eine führt nach der Luftpumpe $L$, die andere zum Vakuumeter $m$. Ist die Luft aus dem Trog entfernt, so läßt man durch den mit Hahn versehenen Trichter $\mathrm{Tr}$, dessen Rohr bis auf den Boden führt, die Gallertflüssigkeit eintreten, bis sie die Filter bedeckt, schließt den Hahn $\mathrm{H}$ zum Trichter und öffnet den $\mathrm{Hahn} \mathrm{H}_{1}$, durch welchen ursprünglich die Luft ausgepumpt wurde; so wird die Gallertflüssigkeit unter Atmosphärendruck in die Filter gepreBt. Nach einiger Zeit nimmt man den Deckel $D$ ab, hebt die Stange $S$ mit den Filtern $F$ aus der Flüssigkeit und läßt abtropfen. Schlieblich gelatiniert man, indem man rasch das ganze Filter in eine geeignete Flüssigkeit taucht; bei Eisessigkollodium genügt Wasser. Arbeitet man mit Gelatine, so muß der ganze Imprägniertrog in einem Bad mit lauem Wasser stehen. Die Härtung der Gelatinefilter erfolgt derart, daß man die an der Luft gelatinierten noch feuchten Filter in eine mit Eis gekühlte 2 bis 4 proz. Formaldehydlösung taucht und einige Tage im Eisschrank stehen läßt.

Die Filter, auf welche Art sie immer gewonnen sein mögen, werden dann mehrere Tage in fließendem Wasser gelassen und in Wasser aufgehoben, dem man etwas Chloroform zusetzt, $\mathrm{mm}$ Schimmelbildung zu unterdrücken.

Es sei hier erwähnt, daß es auch gelingt, durch die üblichen Dyalisiermembranen, wie Pergamentpapier, Fischblase und Amnionha ut zu filtrieren. Unter diesen erwies sich Fischblase als gleichmäßigstes Material. Von einer Lösung von Hämoglobin und von Lackmus in Wasser konnte bei ca. 5 Atmosph. reines Wasser abfiltriert werden, während konzentriertere Lösungen im Filter blieben. Weniger gleichmäBig ist Pergamentpapier. Auch hier beginnt Wasser bei 4 bis $5 \mathrm{Atm}$. zu filtrieren, bei $6 \mathrm{Atm}$. filtrierten ca. $10 \mathrm{ccm}$ in der Stunde. Hämoglobin konnte hier durch Filtration von dem Iösenden Wasser getrennt werden, für Lackmuslösung aber war es ein wenig durchlässig, so daß das Filtrat 1/11 der Konzentration des Filterinhaltes hatte. Im Filter zeigten sich nachher einzelne blaue Punkte, die offenbar undicht waren. Das gleiche Resultat zeigte sich bei Pergamentpapier von zwei verschiedenen Bezugsquellen. Hingegen ließ eine Pergamentmembran selbst bei $7 \mathrm{Atm}$. noch keine Spur einer Ammoniumsulfatlösung durch; sie wirkt offenbar zusammenziehend auf die Membran, während die gleiche Lösung von $5 \mathrm{Atm}$. ab durch eine Fischblase filtriert werden konnte. Uebrigens ist auch Fischblase keineswegs mit den künstlichen Membranen zu vergleichen; obgleich sie erst von 4 bis 5 Atm. ab Flüssigkeit durchläßt, läßt sie noch Deuteroalbumosen, ja sogar teilweise Protalbumosen, filtrieren, die bereits von einem 4 proz. Eisessigkollodiumfilter zurückgehalten wurden, von welchem Wasser schon bei 1,4 Atm. rasch abfiltriert.

Ungemein durchlässig ist die van Calcar'sche 
A mnionmembran, die ich der Liebenswürdigkeit von Herrn Prof. Dr. van Calcar verdanke; sie läßt bereits bei $0,2 \mathrm{Atm}$. Wasser filtrieren, ist aber dann auch stark durchlässig für Lackmus und Hämoglobin.

Die Druckfestigkeit der Pergamentpapiere ist sehr ungleichmäßig; ich hatte solche, die bei 10 Atm. noch dicht waren, während andere schon bei 6 Atm. undicht wurden.

Ganz unbrauchbar für unsere Zwecke sind die diversen Pergamentersatzpapiere, wie Pergamyn etc. Ich habe eine große Zahl untersucht: sie reißen bei 1 Atm. oder weniger. Die künstlichen Membranen sind somit dem Pergamentpapier und den natürlichen Membranen(Fischblase,Amnion) für Filtrationszwecke weit überlegen, da sie trotz ihrer größeren Durchlässigkeit für Wasser eine gleichmäBigere Struktur (Siebwirkung) aufweisen.

Das Wasser läßt sich in den künstlichen Filtern sukzessive durch organische Flüssigkeiten (Alkohol, Aceton etc.) ersetzen, so daB diese Filter auch zur Trennung von und in organischen Lösungsmitteln dienen können. So gelang es z. B. aus einer technischen Chlorophyllösung in Alkohol, die neben Chlorophyll im wesentlichen Fette, Wachse etc. enthält, das Chlorophyll zurückzuhalten, während die Fette etc. das Filter passierten. Alkoholische Mastix-, Seifen-, Tanninlösungen scheinen zum größeren Teil echte Lösungen zu sein, da sie auch sehr dichte Alkoholfilter größtenteils passieren.

\section{Der Filtrierapparat.}

Die Konstruktion eines geeigneten Filtrierapparates machte anfangs einige Schwierigkeiten, da verschiedene Forderungen gleichzeitig $\mathrm{zu}$ erfüllen waren: er mußte unter den verschiedensten Drucken benutzbar, die Filter leicht auswechselbar, die Filterfläche im Verhältnis zum Fassungsvermögen groß sein.

Die beiden Ausführungsarten, welche ich zurzeit benutze, haben sich aufs beste bewährt.2) Das Material ist Rotguß, stark vernickelt.

2) Die Filtrierapparate ebenso wie der Imprägnierapparat und der später erwähnte Ueberführungsapparat sind zu beziehen bei den "Verein. Fabriken für Laboratoriumsbedarf", Berlin N., Chausseestr. 8.
Apparat I (Fig. 2) besteht aus einem zylindrischen Gefäße $\mathrm{H}$, in dem der eigentliche Trichter $\operatorname{Tr}$ aufsitzt. Zwischen die unteren Ausbuchtungen von $\mathrm{Tr}$ und $\mathrm{H}$ werden die runden flachen Filterscheiben $\mathrm{Fi}$ gepreBt. Die Dichtung erfolgt durch zwei Gummiringe GG. Zum Schutz gegen das Reißen des Filters liegt dasselbe auf einem ebenfalls flachen runden Drahtnetz $\mathrm{N}$ auf und ist gegen zu starke Aus- 
dem Fiiter abscheidet und selbst ein neues Filter bildet, das oft dichter ist als das ursprüngliche und abgesehen von Täuschungen eine

große Erschwerung der Filtration zur Folge haben kann. In diesen Fällen hat sich der Rührer als sehr zweckmäBig erwiesen.

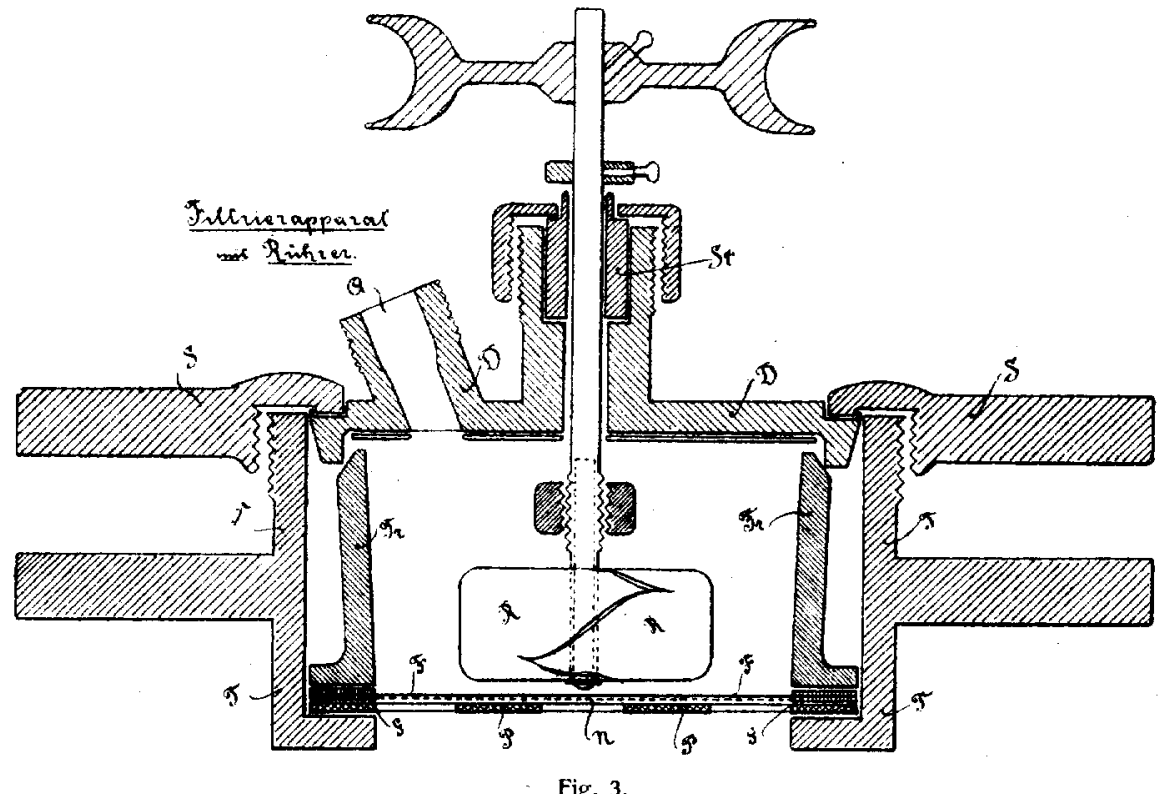

\section{Das Filter.}

Wie bereits erwähnt, nimmt die Porenweite mit der Konzentration der Gallerte $a b$ und es ist demgemäß auch ein höherer Druck erforderlich, um ein Filtrat zu erzielen. Die Zusammensetzung des Filtrats aber ändert sich nicht mit dem Druck sondern nur die Geschwindigkeit der Filtration.

Während die Filter, die aus ein er Herstellung stammen, besonders bei Verwendung von Eisessigkollodium untereinander sehr gleichmäßig sind, weichen die Filter aus verschiedener Herstellung, wenn auch die übrigen Bedingungen die gleichen sind (gleiche Gallertelösung, gleiches Papier etc.) nicht unerheblich voneinander ab. Wahrscheinlich spielte die Temperatur der Imprägnationsflüssigkeit, die Art und Temperatur, bej welcher die Verdünnung der Gallerte vorgenommen wird, und ähnliche, noch nicht näher bestimmte Bedingungen eine wesentliche Rolle: sie beeinflussen unter anderem die Viskosität und damit auch die Dicke der Gallertschicht.

Um nun die Filter verschiedener Herstellung miteinander vergleichen zu können, war es notwendig, einen geeigneten Standard, ein Maß zu suchen, an dem die Durchlässigkeit der Filter gemessen werden konnte. Bis jetzt erwies sich mir am zweckmäßigsten eine 1 proz. Hämoglobinlösung (Hämoglobin in lamellis Merck).
Die größten Poren eines solchen Filters halten noch Teilchen zurück, welche kleiner als $20 \mu \mu$ sind; denn vergleichende ultramikroskopische und Filtrationsversuche mit Hämoglobin und Kollargol haben mir gezeigt, daß Filter, welche eine Kollargollösung von durchschnittlich $20 \ldots u$ gerade vollkommen zurückhalten, nur noch Spuren einer Hämoglobinlösung passieren lassen.

ich bezeichne deshalb jedes Filter nach der Konzentration der Gallerte, mit der es imprägniert ist und gebe in Klammern an, welches Filter (aus der gleichen Herstellung) 1 prozentige Hämoglobinlösung, die mindestens zwei Tage gestanden hat, gerade zurückhält ${ }^{3}$ ); z. B. 3 Proz. (H 4 Proz.) heißt: ich habe ein Filter, das mit 3 proz. Gallertlösung getränkt ist; es läßt Hämoglobinlösung reichlich passieren, da diese erst von einem Filter gleicher Herstellung vollständig zurückgehalten wird, die mit 4 proz. Gallerte getränkt ist. Ganz andere Eigenschaften hat ein Filter 3 Proz. (H 2,5 Proz.). Dies ist ebenfalls mit 3 proz. Gallerte getränkt, läßt aber kein Hämoglobin mehr durch, denn dies wird

3) Anm. Nach J. Lemanissier (Études des corps ultra-microscopiques, Paris) zeigen Hämoglobinlösungen anfangs im Ultramikroskop Submikronen, nach $48 \mathrm{~h}$ aber beinahe gar keine Teilchen mehr. Aehnliches zeigen Glykogenlösungen (Raehlmann, Gatin-Gruzewska u. Biltz). Die gröberen Verteilungen gehen sonach mit der Zeit in Lösung. 
schon von einem mit 2,5 proz. Gallerte getränktển Filter zurückgehalten. Die meisten späteren benutzten Filter sind im Vakuum imprägniert; sie sind mit v bezeichnet z. B. 3 Proz. v (H 2,5 Proz.). Bei Gelatinefiltern ist noch die Konzentration der Formaldehydlösung beigefügt z. B. G 5 Proz. v F 1 Proz. (H, G 4 Proz. v F 1 Proz) d. $h$. es ist ein Gelatinefilter mit 5 Proz. Gelatinelösung im Vakuum imprägniert und in 1 proz. Formaldehydlösung gehärtet; es ist für Hämoglobinlösung undurchlässig, die gerade durch ein gleich behandeltes 4 proz. Gelatinefilter zurückgehalten wird.

Bei allen Versuchen und Messungen, bei welchen nichts besonderes bemerkt wird, wurde nur eine Filterscheibe benutzt. Man kann natürlich dem Filter eine größere Dichte geben, indem man mehrere Scheiben gleicher oder verschiedener Dichte übereinander legt, was für praktische Zwecke zuweilen von Vorteil ist.

Um eine Vorstellung zu geben, welche Drucke bei der Filtration in Betracht kommen, seien einige Zahlen aus meinen Protokollen angeführt, die einerseits zeigen, mit wie geringen Ueberdrucken - alle Zahlen bezeichnen Ueberdrucke über $1 \mathrm{Atm}$. - man bei den weitporigen Filtern auskommt, wie sehr aber der Druck bei den dichteren Filtern gesteigert werden muß und welchen Einfluß das Wasseranziehungsvermögen (Hydrophylie) des gelösten Kolloids (z. B. Serum, Magermilch) auf die Filtrationsgeschwindigkeit hat. (Alles ist auf eine Minute umgerechnet.) Es filtrierten bei einer Filterfläche von ca. $35 \mathrm{qcm}$ durch ein

\section{Filtrat Filtrans}

\begin{tabular}{|c|c|c|c|c|c|c|c|c|c|c|}
\hline 2,1 proz. & Eisessigkollodium & bei & 0,5 & Atm. & in & 1 Min. & 24 & $\mathrm{ccm}$ & (unreines & Wasser) \\
\hline 2,1 & " & $n$ & 1 & " & ” & 1 & 40 & " & " & " \\
\hline 2,5 & $"$ & $"$ & 0,5 & $"$ & $"$ & $1 \%$ & 8,3 & $"$ & " & " \\
\hline 2,5 & $"$ & $"$ & 1,0 & $"$ & $"$ & $1 "$ & 16,6 & $n$ & \multirow{5}{*}{\multicolumn{2}{|c|}{$\begin{array}{l}\text { (Magermilch)" } \\
\text { (koll. Eisenoxyd) } \\
\text { (Vollserum) } \\
\text { (0,5 Proz. lysargins. Na) }\end{array}$}} \\
\hline 2,5 & $n$ & $"$ & 1,5 & $"$ & $"$ & 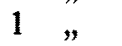 & 0,5 & $"$ & & \\
\hline$"$ & $"$ & 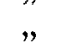 & 1,0 & 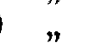 & $\eta$ & $"$ & 3,5 & $"$ & & \\
\hline 4,5 & " & " & 2 & ” & ” & $"$ & 2 & $"$ & & \\
\hline$"$ & $n$ & , & 0,5 & " & $"$ & $"$ & 0,1 & $"$ & & \\
\hline$"$ & $"$ & $"$ & 2 & , & $\eta$ & $"$ & 0,28 & 8 & \multirow{4}{*}{\multicolumn{2}{|c|}{$\begin{array}{l}\text { (verdünnte } \\
\text { Kollargollösung) }\end{array}$}} \\
\hline$"$ & $"$ & $"$ & 5 & $"$ & 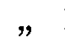 & $"$ & 0,65 & 5 & & \\
\hline " & $"$ & $"$ & 8 & $"$ & $"$ & $"$ & 1 & ” & & \\
\hline$\because$ & " & & 10 & & & & 1,1 & & & \\
\hline
\end{tabular}

Die Filterwirkung.

Es genügt jedoch nicht nur, die Zusammensetzung des Filtrats zu ermitteln. In vielen Fällen muß man sich überzeugen, ob im glvichen $\mathrm{MaB}$, in dem sich das Filtrat verdünnt hat, auch

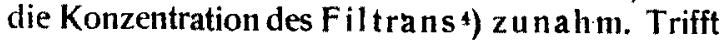
dies nicht zu, erweist sich, daß das. Filtrans nicht konzentrierter oder gar verdünnter ist, wie die Originallösung, so kann man mit Sicherheit annehmen, daß das Filter den gelösten Stoff der Lösung entzogen, ihn adsorbiert hat. In solchen Fällen ist von einer Filterwirkung nicht mehr die Rede; man kann dann die Rolle der Teilchengröße nur dadurch ermitteln, daß man so große Mengen der Lösung durch das Filter schickt, daß dieses gesättigt ist.

Leider versagt diese Möglichkeit häufig in der Praxis. In der Biologie und Medizin hat man es viel mit Fermenten und Toxinen zu tun; man hat meist sehr kleine Substanzmengen mit enormer Wirkung vor sich. Gerade die Fermente und Toxine besitzen jedoch, im Gegen-

t) So bezeichne ich die Flüssigkeit, welche filtriert werden soll. satz zu den ziemlich indifferenten Eiweißkörpern, eine enorme Verwandtschaft zu den verschieVerwandschaft von Farbstoff zur Faser vergleichen läBt. Das Filter entzieht fast unbegrenzte Mengen aus der Lösung, so daß die Porenwirkung kaum zur Geltung kommt.

Ich habe früher den Ausdruck "Siebwirkung " gebraucht; daraus, sowie aus den vorigen Darlegungen könnte leicht eine mißverständliche Auffassung über die Wirkung dieser Filter entstehen. Bei einem Sieb ist ein Loch so weit wie das andere. Hat man Schrot von gleicher Korngröße, so fällt eventuell jedes Korn durch jedes Loch. Aber offenbar sind die Poren eines Filters ungleichmäBig grof, sie lassen sich weit eher mit denen eines Schwammes vergleichen, ebenso wie die Teilchen einer kolloiden Lösung bestimmter Konzentration untereinander mehr oder minder verschieden sind.

\section{Die Ungleichheit der Filterporen}

läßt sich leicht durch Augenschein wahrnehmen. Filtriert man eine gefärbte kolloide Lösung, z. B. Kollargol, durch eine Serie vón Filtern denen Filtermaterialien, die sich nur mit der 
verschiedener Dichte z. B. 1,5 Proz.; 2,5 Proz.; 3,5 Proz.; 4 Proz.; 4,5 Proz.; 5 Proz., so geht die Lösung durch das 1,5 Proz. Filter noch fast unverändert durch, das Filter ist beiderseits tiefschwarz gefärbt; durch das 2,5 Proz. Filter läuft noch eine tiefbraune, durch das 3,5 Proz. Filter eine hellgelbe, durch das 4 Proz. Filter eine ganz schwach gefärbte Lösung und durch das 4,5 Proz. und 5 Proz. Filter läuft scheinbar klares Wasser. Sämtliche Filter sind auf der Filtransseite tiefschwarz. Auf der Filtratseite ist das 2,5 Proz. Filter dunkel gefärbt, mit helleren Partien; das 3,5 Proz. Filter zeigt eine größere Anzahl dunkler Flecken in weikem Feld; das 4 Proz. Filter zeigt noch einzelne dunkle Punkte, das 4,5 Proz. Filter immer noch vereinzelte dunkle Pünktchen und das 5 Proz. Filter ist auf der Filtratseite ganz weiß. Analoges kann man bei allen gefärbten kolloiden Lösungen, bei Berlinerblau, kolloidem Eisenoxyd etc. beobachten. Die Zahl der für die kolloide Lösung durchlässigen Stellen nimmt somit bei zunehmender Filterdichte $a b$.

$\mathrm{DaB}$ auch die Teilchen einer Lösung unter sich nicht gleich groß zu sein pflegen, ergeben die ultramikroskopischen Untersuchungen von $Z$ sigmondy, denen sich analoge Resultate von Biltz und Raehlmann anschlossen.

Auch die Filtrationsmethode bot eine Handhabe, Verschiedenheiten in der Teilchengröße des gleichen Kolloids nachzuweisen und zur Trennung beizutragen.

lch habe eine Kollargollösung eine Stunde lang bei 6000 Umdrehungen in der Minute zentrifugiert und erhielt so drei Fraktionen. Auf dem Boden hatte sich eine schwarze Masse abgesetzt, von dieser war ein Teil A unlöslich in Wasser, der andere Teil B löslich in Wasser, sehr stark opaleszierend. Im Ultramikroskop sah man hauptsächlich sehr helle gelbe, mäßig bewegliche Punkte. Auf dem Gallert-Filter setzte diese Fraktion einen rostroten Niederschlag $a b$, während sonst der Rückstand von einer Kollargol-Filtration tiefschwarz metallisch glänzend ist. Auch die stark verdünnte Lösung war rotstichig. Die beim Zentrifugieren gelöst gebliebene Fraktion $C$ war sehr wenig opaleszierend und zeigte im Ultramikroskop lauptsächlich grüne, ziemlich lichtschwache, sehr bewegliche Punkte. Auf dem Filter setzte sich der übliche metallglänzende Kollargolniederschlag $a b$ und die stark verdünnte Lösung war braungelb. - Das verschiedene Absetzen beim Zentrifugieren, sowie die verschiedene Beweglichkeit und Lichtstärke in Ultramikroskop erweisen die verschiedene Teilchengröße in jener Kollargollösung. Ich konnte nun ein Filter ausfindig machen, das die Kollargolfraktion $\mathrm{C}$ zum großen Teil passieren ließ, während es die Kollargolfraktion B zum größten Teil zurückhielt.

\section{Die Teilchengröße.}

Die rein mechanische Vorstellung muB zu der Ueberzeugung führen, daß es möglich ist, durch zunehmende Dichtung eirtes Filters immer feinere Partikel zurückzuhalten. Auf diesem Prinzip sind die beschriebenen Filter hergestellt und auf Grund der damit vorgenommenen Filtrationen habe ich nachstehende Tabelle aufgestellt, welche von oben nach unten die zunehmende Kleinheit der Teilchen in den untersuchten Lösungen erweisen soll. Sie reicht bis gegen $H 4$ Proz. $v$ in das noch der ultramikroskopischen Untersuchung zugängliche Gebiet von 1 bis $4 \mu \mu$. Innerhalb dieser Grenze zeigt sich im ganzen eine Uebereinstimmung mit den Ergebnissen der ultramikroskopischen Beobachtung. Volle Uebereinstimmung wird durch Literaturvergleich überhaupt nicht $z u$ erreichen sein, denn Biltz, Zsigmondy u. a. haben gezeigt, daß die Teilchengröße bei anorganischen Kolloiden in erster Linie von der Herstellungsweise des Sols abhängt und beliebig variiert werden kann. Während z. B. kolloides Berlinerblau wegen seiner grob sichtbaren Teilchen ein beliebtes Demonstrationsobjekt am Ultramikroskop ist und auch bei mir als gröbste kolloide Lösung figuriert, konnte $\mathrm{Biltz}$ durch geeignete Modifikationen ein kolloides Berlinerblau herstellen, das nur aus Amikronen bestand. Ich habe deshalb vermittelst des Ultramikroskops feste Anhaltspunkte zu gewinnen versucht. So sei vorausgeschickt, daB sich durch Filtratior. aus gewöhnlichem destillierten Wasser durch meine Filter ein sehr brauchbares Wasser für ultramikroskopische Zwecke gewinnen läßt.

Ich bestimmte die Teilchendurchmesser in einer frischen Kollargollösung zu durchschnittich $20 \mu \mu$. Da Kollargol von Filtern gerade zurückgehalten wird, die auch Hämoglobin gerade zurückhalten oder nur wenig durchlassen, so sind die größten Poren des $\mathrm{H}_{4}$ Proz. v Filter etwas $20 \mu \mu$.

Ich verdanke ferner der Liebenswürdigkeit von Herrn Dr. Zsigm ondy zwei Goldlösungen, deren Teilchengröße er selbst bestimmt hatte. Ein Filter, welches die Goldlösung mit ca. $40 \mu \mu$ vollkommen zurückgehalten hatte, ließ von der Lösung mit Teilchen von ca. $4 \mu \mu$ noch einen Teil passieren. Auch der von mir vorher mit- 
geteilte Versuch mit den beiden zentrifugierten Kollargolfraktionen ist beweisend dafür, da $\mathrm{B}$ in der Tat mit zunehmender Filterdichte immer kleinere Teilchen zurückgehalten werden.

Ein ganz einheitliches Bild ist von nachstehender Tabelle nicht zu erwarten, weil sie ja nur die untersten Grenzen der Durch. lässigkeit bezeichnet: besteht eine Lösung zumeist aus groben Teilchen und nur aus einer Minderheit von ganz kleinen Teilchen, so wird sie dnch in gleicher Reihe stehen mit einer Lösung, die nur solch ganz kleine Teilchen enthält.

Die Angaben beziehen sich auf Filter, die gerade die betreffenden Lösungen vollkommen zurückhalten.

Suspensionen.

2 Proz. v (H 4 Proz. v) Berlinerblau (+ lysalbinsaures $\mathrm{Na}$ )

2 " v (H 4 , v) Platinsol (nach Bredig)

2 " v (H 4 " v) Kolloides Eisenoxyd

2,5 " $(\mathrm{H} 4$, v) Kasein (in Milch)

3 " v (H 4 ” v) Kolloides Arsensulfid (+ lysalbinsaures $\mathrm{Na})$

3 " v ( $\mathrm{H}_{4}$, v) Goldlös. (Zsigmondy) + lysalbins. Na Nr. 4 (ca. $40, \mu \mu$ )

3 v (H 4 , v) Bismon (koll. Wismutoxyd n. Paal)

$3,5, v(\mathrm{H} 4$, v) Lysargin (koll. Silber n. ('aal)

3,5 " v bis 3,5 Proz. (H 4 Proz. v) Kollargol Silber v. Heyden (kolloid. ca. $20 \mathrm{mu})$

3,5 „ v (H 4 Proz. v) Goldlös. (Zsigmondy) + lysalbins. Na Nr. 0 $\left.(\text { ca. } 4, \mu \mu)^{5}\right)$

4 " v (H 4 , v) 1 Proz. Hämoglobinlösung

$4 \quad$ v ( 4 " v) 1 proz. Gelatinelösung

4 " v bis 4,5 Proz. (H 4 Proz. v) Serumalbumin (Molekulargew. 5000 bis 15000 )

5) Die Stellung dieser Goldlösung in ihrem Verhältnis zu Hämoglobin kann noch nicht als definitiv angesehen werden. etwas über 4 Proz. v (H 4 Proz. v) Diphtherietoxin

$4,5, \quad v(\mathrm{H} 4$, v) Protalbumosen Kieselsảure

8 " v ( 4 \# v) Deuteroalbumosen

A (Molekulargewicht ca. 2400)

10 " v (H 4 , v) passiert Spuren von Deuteroalbumosen B 8. C Lackmus

$10 " \mathrm{v}(\mathrm{H} 4, \quad$ v) passiert in kleinen Mengen Dextrin (Molekulargew. ca. 965) Kristalloide.

Trotz angeführten Bedenken sehen wir doch, daß die nach der Filtrationsmethode gruppierte Reihenfolge in der Teilchengröße der untersuchten Stoffe übereinstimmt mit dem, was wir durch theoretische Ueberlegung und Ultramikroskop von ihnen wissen:

Wir sehen, daß die nach den gewöhnlichen Vorschriften hergestellten annrganischen Kolloide die gröbste Zerteilung aufweisen, daß eine Goldlösung von $40 \mu \mu$ von einem gröberen Filter zurückgehalten wird, als eine von ca. $4 \mu \mu$; sowie, daß koll. Metallösungen, die in Gegenwart eines Schutzkolloids hergestellt sind, (Kollargol, Lysargin) eine feinere Zerteilung aufweisen als ohne Schutzkolloid (Platinsol).

Die organischen Kolloide wie Serumalhunin (Molekulargewicht 5000 bis 15000) und Hämoglobin zeigen nach der Filtrationsmethode beurteilt ein gröberes Korn als ihre Spaltungs. produkte, die Albumosen (Molekulargew. ca. 2400) und das Hämatin. Unter den Albumosen zeigen sich Verschiedenheiten in der Filtrierbarkeit entsprechend ihrer Salzfällbarkeit, dip ja mit gröBter Wahrscheinlichkeit von der Teilchengröllte abhängt. Durch noch engere Filter passiert das Dextrin, dem man das Molekulargewicht 965 zuschreibt und die Kristalloide.

Wir sehen somit alle Voraussetzungen erfüllt und glauben behaupten zu dürfen, daß die beschriebene Filtrationsmethode eine Scheidung nach der Teilchengröße gestattet und daB ihre Anwendbarkeit schon jetzt weit in das Gebiet der Amikronen herabreicht, bis zu Körpern, die man bereits zu den Kristalloiden zählen kann.

(Fortsetzung u. Schluß im nächsten Hefte.) 